\title{
TWO-PHASE ACTUATORS: STABLE ZIPPING DEVICES WITHOUT FABRICATION OF CURVED STRUCTURES
}

\author{
J.R. Gilbert and S.D. Senturia \\ Microsystems Technology Laboratories, MIT, Cambridge MA 02139
}

\begin{abstract}
The design of electrostatic actuators optimized for both high force generation as well as long and stable displacement is difficult. Several groups have proposed and/or implemented devices based on "zipping" to achieve improvements in both areas simultaneously. In these designs the implementation of a zipping actuator requires the fabrication of a curved surface on which one electrode touches another. The fabrication of controlled curved surfaces often presents difficulties, especially those perpendicular to the wafer surface. In this paper we propose and model a class of devices, two-phase actuators, which can obtain similar effects without need for fabrication of curved structures.
\end{abstract}

\section{INTRODUCTION}

Practical use of electrostatic actuation often encounters two difficulties: (1) the electrostatic force scales as $1 / x$, giving very short displacement if large forces are needed, and (2) electrostatic spring-mass systems exhibit pull-in instabilities and so have stable controllability only over a fraction of their travel. Much of the design of electrostatic actuators is devoted to working around or utilizing these two effects. Several groups have proposed and/or implemented devices based on "zipping" to overcome both of these effects $[1,2,3,4]$. In these designs the implementation of a "zipping" actuator with stable motion requires the fabrication of a curved surface on which one electrode touches another. We propose a class of devices, twophase actuators, which can obtain similar effects without the need for fabrication of curved structures. This design concept emerged in our work on MEMCAD and the CoSolve-EM package for 3D simulation of coupled electromechanics[5] and contact electromechanics[6]. In this paper we use those tools to simulate a voltage controlled torsion mirror (VCM) as a representative example of this class of devices.

\section{ZIPPING ACTUATORS}

The simplest conceptual design of a zipping actuator is given by Branedbjerg. An S-shaped flexible electrode is positioned next to a flat ground plane and separated from it by a thin dielectric layer. Such a device is sketched in Figure 1. The flexible electrode has one end clamped high and the other end clamped low, near the ground layer. The device has an Sshaped transition region from its high end to its low end. A voltage is applied between the electrode and ground, and the transition region moves from right to left. This motion of the transition region is one measure of the "displacement" of the device. Because the electrostatic loads on the structure vary as $1 / x$, where $x$ is the distance between the electrode and ground, the circled region in Figure $1 \mathrm{~B}$ gives the dominant contribution to the driving force. The shape of this driving region is essentially maintained as the transition region moves from right to left, and so the driving force is nearly constant as the transition region moves.

Most zipping actuators share these two qualitative features: a curved transition region that is displaced under operation, and a region of close apposition of the conductors that provides the dominant contribution to the driving force of the device.

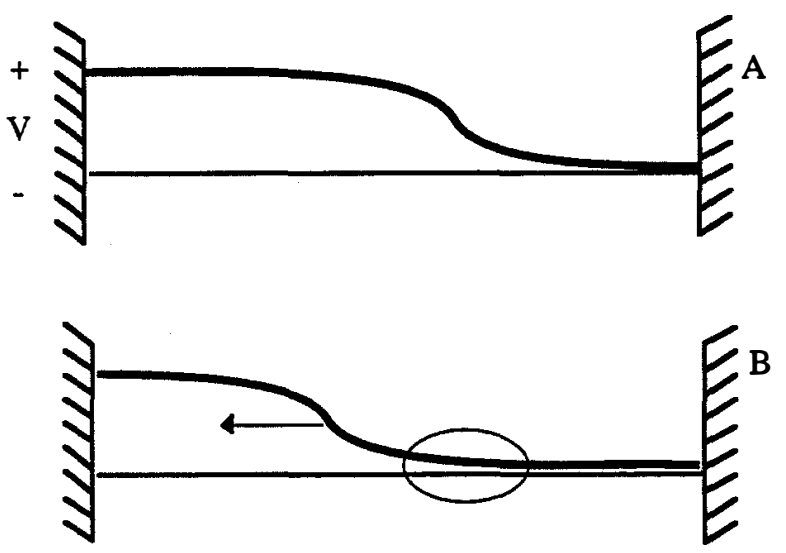

Figure 1 A Schematic example of a "zipping" actuator. Increasing the voltage $V$ drives the mid-point of the " $S$ " to the left. In all states of the actuator its driving force is dominated by the region where the electrode and ground are most closely in contact, but not yet touching-this region is circled in $(B)$. The dielectric layer over the ground plane is not shown.

\section{TWO-PHASE ACTUATOR DESIGN}

The two-phase (or multiple-phase) actuator concept is to produce the curved moving electrode by the operation of a "starter" structure, instead of fabricating that curve directly.

A proto-typical two-phase actuator has one movable conducting part, the "plate", and two or more fixed electrodes. Either the plate or both fixed electrodes are insulated to prevent shorts upon contact. The plate is held at some fixed voltage $V_{F}$. One electrode, the "starter", deforms the plate into initial contact using a voltage, $\mathrm{V}_{s}$. The other electrode, the "driver", is then in close contact with the plate, and may drive it through stable zipping motion by ramping its drive voltage, $V_{D}$, from 0 to some $V_{\text {MAX }}$. As an example of such a two phase actuator we will discuss a voltage controlled mirror (VCM). Figure 2 shows a 3D view of the VCM. In Figure 3 its dimensions are sketched. The "plate" is modeled in this study as aluminum, with tensile residual stress of $40 \mathrm{MPa}$. The plate has a thick mirror region, and a long thin neck. At the end of the neck there is a wider area used to reduce the initial starting voltage. The mirror region is suspended on two tethers which are labeled in Figure 2. These 
tethers are purposely set off center in the mirror to allow the mirror to tilt to almost 40 degrees, before it will touch down at the point where it joins the neck. There is an insulating contact layer $0.05 \mu \mathrm{m}$ above the Driver and Starter electrodes. In operation the thickness of this separation layer determines the scale of the driving force of the device.

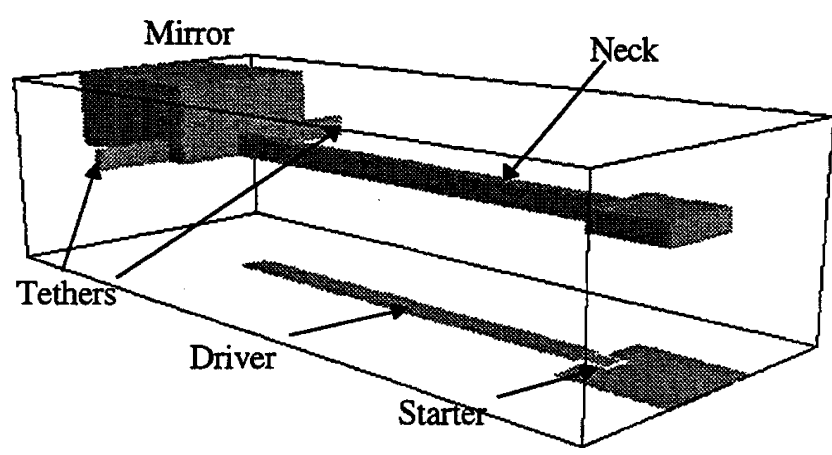

Figure 2 A $3 D$ view of the VCM structure. The "Plate", with labeled regions: Mirror, Neck, and Tethers, is $3.0 \mu \mathrm{m}$ above the Driver and Starter electrodes. There is an insulating contact layer $0.05 \mu \mathrm{m}$ above the Driver and Starter electrodes.
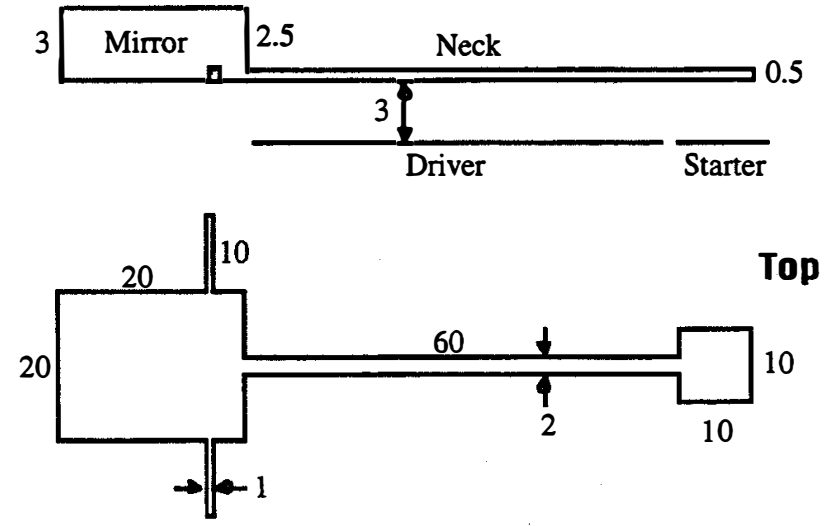

Figure 3 Top and Side views of the VCM. All numbers in $\mu m$.

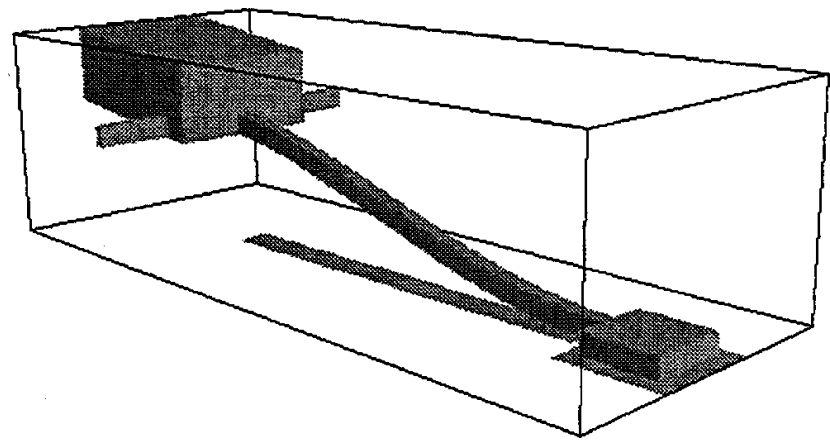

Figure 4 The Model at drive voltage $V_{D}=0$. The starter has pulled in the mirror plate, establishing the basic curve for the operation of the VCM. The mirror has an initial tilt angle of 1.4 degrees.

\section{TWO-PHASE ACTUATOR SIMULATION}

We built a detailed 3D model of the VCM, using the MEMCAD system. Its quasi-static behavior was then simulated using CoSolve-EM v1.0.

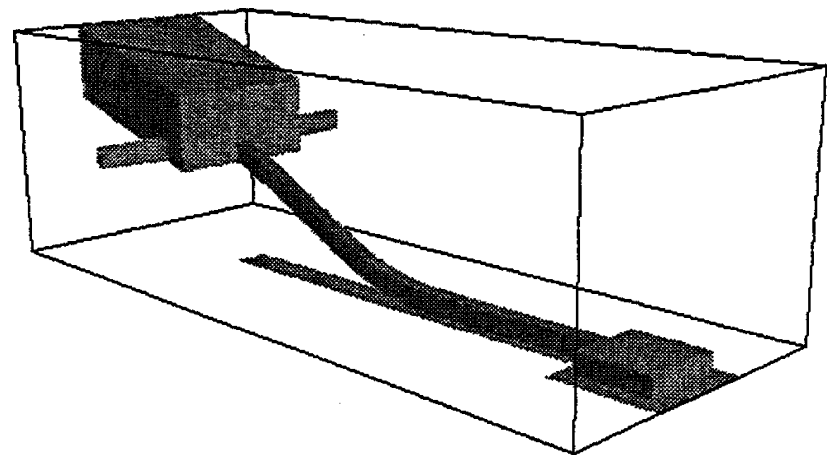

Figure 5 The Model at drive voltage $V_{D}=20 \mathrm{~V}$. The drive is zipping in the neck of the mirror plate, causing the mirror to tilt. The tilt angle is 3.5 degrees.

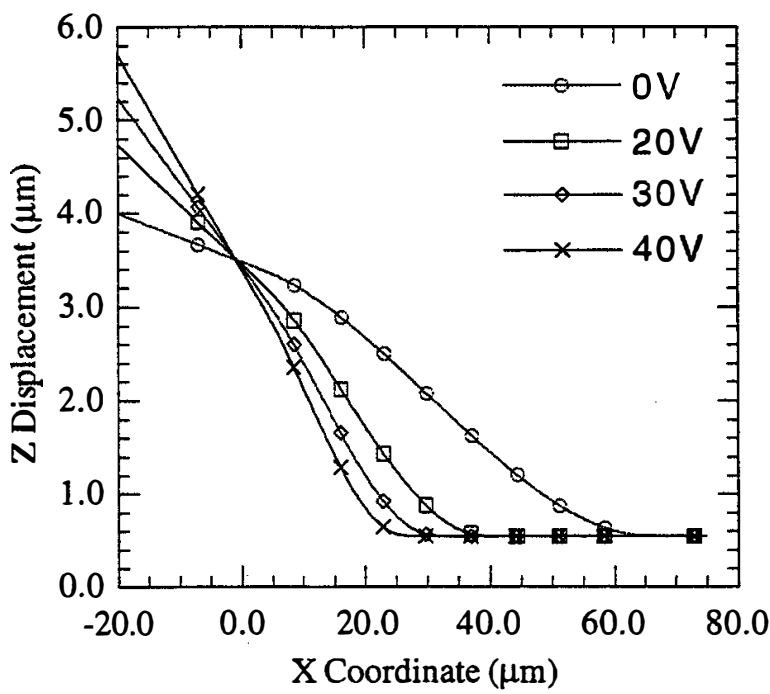

Figure $6 X-Z$ cross-sections through $y=0$ on the bottom surface of the plate at four different $V_{D}$. The mirror itself extends from $-20 \mu \mathrm{m}$ to $+4 \mu \mathrm{m}$ and rotates rigidly as the device zips in. The cross-section of the undisplaced surface is constant at $z=3.5 \mu \mathrm{m}$.

In simulation we apply $\mathrm{V}_{s}=20 \mathrm{~V}$ to the starter electrode, and the long neck of the plate pulls in to its initial operating position, as shown in Figure 4. A curved shape has now been established by electrostatic actuation and the driver electrode is in contact with the plate at the location nearest the starter. The shape in Figure 4 is considered the initial operating state of the VCM. In operation we control the driving voltage $V_{D}$. As we ramp $V_{D}$ from $0-80 \mathrm{~V}$ the neck of the movable conductor zips in, causing the mirror to tilt. Figure 5 shows the VCM at $V_{D}=20 \mathrm{~V}$. The transition region of the zipper has moved towards the mirror. Figure 6 plots cross-sections along the length of the 
bottom surface of the plate at four drive voltages, $0 \mathrm{~V}, 20 \mathrm{~V}$, $30 \mathrm{~V}$, and $40 \mathrm{~V}$. These cross-sections show the transition region moving in towards the mirror, and the mirror tilting, while remaining undistorted. Figure 7 shows the dependence of mirror tilt angle on $V_{D}$ over the operating region of the $V C M, V_{D}=0$ $80 \mathrm{~V}$, for which $\phi=1.4-10.3$ degrees. The angular dependence is surprisingly linear for so complex a structure. The mirror is designed to tilt up to 40 degrees, though we have not yet simulated it that far. In a more standard electrostatic torsion mirror design, allowing for the possibility of such high angles would substantially reduce the available area and force for driving the mirror. The VCM is stable over its operating region: that is, the mirror exhibits no hysteresis. This is a distinct advantage of operation in the zipping mode, essentially beyond the pull-in point. A more standard torsional micromirror might have stable motion only above pull-in, over approximately one third of its range of tilt.

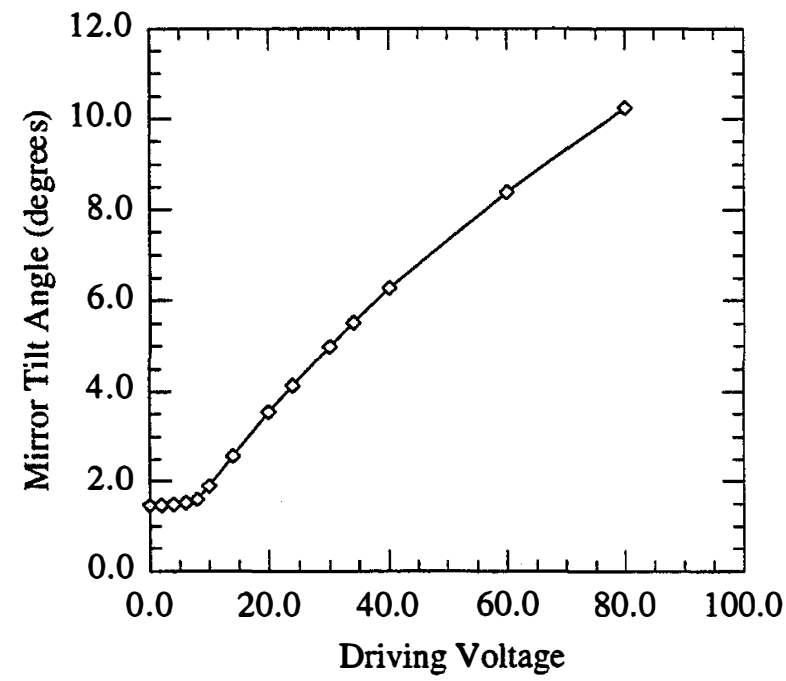

Figure 7 Driving voltage $V_{D}$ vs. the mirror tilt angle. We obtain near linear control of tilt angle over most of the VCM's operating range of 1.4-10.3 degrees.

\section{CONCLUSION}

We have proposed a class of electromechanical actuators, two-phase actuators, that have the benefits of zipper actuators, without the need for fabrication of controlled curved surfaces. We have simulated one particular example of a two-phase actuator, the Voltage Controlled Mirror (VCM). The VCM that is simulated here is compatible with fabrication by surface micromachining techniques. The underlying concept, however, is broadly applicable to a variety of MEMS fabrication technologies. The VCM exhibits continuous stable control of its tilt angle over a full 10 degrees, a result which is difficult to duplicate in a surface micromachined process, if the mirror must operate above pull-in. The modeling and simulations of the VCM were done using the MEMCAD system. This work is also a demonstration of the capability to do complex design and analysis tasks in advance of fabrication using MEMCAD.

\section{ACKNOWLEDGMENTS}

This work has been supported in part by DARPA contract J92-FBI-196 and by equipment grants from Hewlett Packard and Digital Equipment. The authors would like to thank Rob Legtenberg for many valuable discussions and insights.

1 Branedbjerg and Gravesen, "A New Electrostatic Actuator Providing Improved Stroke Length and Force", Proc. MEMS 1992, Travemünde, Germany, pp. 6-11.

2 K. Sato and M. Shikida, "Electrostatic Film Actuator with a Large Vertical Displacement", Proc. MEMS 1992, Travemünde, Germany, pp. 1-5.

3 R. Legtenberg, et al., "Electrostatic Curved Electrode Actuator", Proc. MEMS 1995, Amsterdam, pp. 37-42.

4 M. Yamaguchi, S. Kawamura, K. Minami, and M. Esashi, "Distributed Electrostatic Micro Actuator", Proc. MEMS 1993, Ft. Lauderdale, pp. 18-23.

5 J.R. Gilbert, R. Legtenberg, and S.D. Senturia, "3D Coupled Electro-Mechanics for MEMS: Applications of CoSolve-EM", Proc. MEMS 1995, Amsterdam, pp. 122127.

6 J.R. Gilbert, G.K. Ananthasuresh, and S.D. Senturia, "3D Modeling of Contact Problems and Hysteresis in Coupled Electro-Mechanics", Proc MEMS 1996, San Diego. 